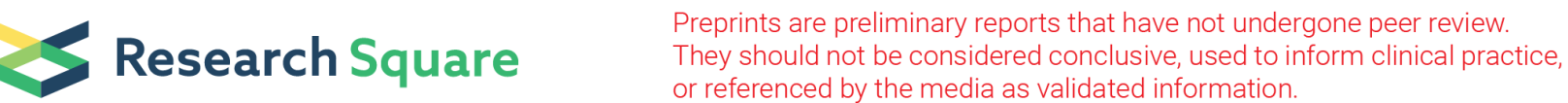

\section{Comparison of Antimicrobial Resistant Neisseria gonorrhea to Ceftriaxone and Ciprofloxacin using Partial Proportional Odds Model for patients seen at the University Teaching Hospital in Lusaka, Zambia}

PRISCILLA KAPOMBE ( $\nabla$ priscilla.kapombe@gmail.com )

University of Zambia School of Medicine https://orcid.org/0000-0003-3914-8873

LUNGOWE SITALI

University of Zambia

PATRICK MUSONDA

University of Zambia

Research article

Keywords: Antimicrobial Resistance, Neisseria gonorrhea, Ciprofloxacin, Ceftriaxone, Partial Proportional Odds Model, Ordinal Logistic Regression, Syndromic treatment

Posted Date: July 15th, 2019

DOI: https://doi.org/10.21203/rs.2.11348/v1

License: (c) (1) This work is licensed under a Creative Commons Attribution 4.0 International License.

Read Full License 


\section{Abstract}

Background Neisseria gonorrhea, the causative agent of Gonorrhea, has developed antibiotic resistance to the "last-line" Cephalosporin's, Quinolones and Macrolides which is cause for concern. In Zambia, despite recommendations of discontinued use, Ciprofloxacin is used for treatment. The lack of an active surveillance system, appropriate and structured data management and analysis tools magnifies the problem because resistance patterns cannot be monitored. The study aimed to ascertain effectiveness of Ciprofloxacin a Quinolone in comparison with Ceftriaxone a Cephalosporin, in treatment of Gonorrhea using Susceptibility testing; and to identify possible risk factors associated with resistance. Methods Study design was parallel non-inferior quasi experimental study. Patients at the University Teaching Hospital with discharge and Gonorrhea symptoms who gave consent, were recruited. Fishers Exact Test for associations was used. Data was analyzed using Ordinal logistic regression as the Susceptibility was at 3 levels; Susceptible, Intermediate or Resistant with an assumed Ordinal nature. Proportionality assumption was checked, and when violated Partial Proportional Odds Model was used instead. Results A total of 104 isolates were obtained. The overall proportion of patients who had Susceptible, Intermediate and Resistant results were: 49 (47.1\%), 55 (52.9\%) and 0 (0) for Ceftriaxone and 70 (68.0\%), 10 (9.7\%) and 22 (22.3\%) for Ciprofloxacin respectively. Adjusted estimates in partial Proportional Odds model showed that, Males were $4.1(95 \% \mathrm{Cl} ; 1.8,9.4$, p-value=0.001) times more likely to have Intermediate or Susceptible results compared to Resistance than females, or they were more likely to have Susceptible compared to Resistance or Intermediate result compared to females. Ciprofloxacin was $70 \%$ less likely than Ceftriaxone of having susceptible or intermediate results compared to resistance and this could be as high as $90 \%$ and as high as $40 \%$ p-values $<0.001$ ). Conclusion Level of Ciprofloxacin resistance detected from the Susceptibility testing, shows it is not an effective treatment for Gonorrhea. Ceftriaxone remains a satisfactory option for first-line treatment of Gonorrhea at UTH. Risk factors identified to be associated with resistance in this study were being female and use of Ciprofloxacin. Ethical Clearance Ethical clearance was obtained from University of Zambia Biomedical Ethics Research Committee (UNZABREC Ref \# 033-06-17).

\section{Abstract}

Background: Neisseria gonorrhea, the causative agent of Gonorrhea, has developed antibiotic resistance to the "last-line" Cephalosporin's, Quinolones and Macrolides which is cause for concern. In Zambia, despite recommendations of discontinued use, Ciprofloxacin is used for treatment. The lack of an active surveillance system, appropriate and structured data management and analysis tools magnifies the problem because resistance patterns cannot be monitored. The study aimed to ascertain effectiveness of Ciprofloxacin a Quinolone in comparison with Ceftriaxone a Cephalosporin, in treatment of Gonorrhea using Susceptibility testing; and to identify possible risk factors associated with resistance.

Methods: Study design was parallel non-inferior quasi experimental study. Patients at the University Teaching Hospital with discharge and Gonorrhea symptoms who gave consent, were recruited. Fishers Exact Test for associations was used. Data was analyzed using Ordinal logistic regression as the 
Susceptibility was at 3 levels; Susceptible, Intermediate or Resistant with an assumed Ordinal nature. Proportionality assumption was checked, and when violated Partial Proportional Odds Model was used instead.

Results: A total of 104 isolates were obtained. The overall proportion of patients who had Susceptible, Intermediate and Resistant results were: 49 (47.1\%), 55 (52.9\%) and 0 (0) for Ceftriaxone and 70 (68.0\%), $10(9.7 \%)$ and 22 (22.3\%) for Ciprofloxacin respectively. Adjusted estimates in partial Proportional Odds model showed that, Males were 4.1 (95\% Cl; 1.8, 9.4, p-value=0.001) times more likely to have Intermediate or Susceptible results compared to Resistance than females, or they were more likely to have Susceptible compared to Resistance or Intermediate result compared to females. Ciprofloxacin was $70 \%$ less likely than Ceftriaxone of having susceptible or intermediate results compared to resistance and this could be as high as $90 \%$ and as high as $40 \%$ p-values $<0.001)$.

Conclusion: Level of Ciprofloxacin resistance detected from the Susceptibility testing, shows it is not an effective treatment for Gonorrhea. Ceftriaxone remains a satisfactory option for first-line treatment of Gonorrhea at UTH. Risk factors identified to be associated with resistance in this study were being female and use of Ciprofloxacin.

Keywords: Antimicrobial Resistance, Neisseria gonorrhea, Ciprofloxacin, Ceftriaxone, Partial Proportional Odds Model, Ordinal Logistic Regression, Syndromic treatment.

\section{Background}

Gonorrhea is a major public health concern globally that requires immediate international public health resources and attention. The World Health Organization reports [1] that Gonococci infections represent 106 million of the estimated 498 million new cases of curable STIs that occur globally every year. Neisseria gonorrhea, the causative agent of Gonorrhea, has developed antibiotic resistance to the "lastline" Cephalosporin's, Quinolones and Macrolides which is cause for concern. Gonorrhea is grossly underreported, particularly in areas where the incidence is high. Accurate and reliable incidence and prevalence data are essential to define the true scope of the problem, to monitor the success of intervention programs, and to provide a basis for informed advocacy. While improving global estimates of the prevalence and incidence of STIs will require concerted efforts by international agencies, national health services, private companies, and the research community [2].

Antibiotic resistant $N$. gonorrhea is emerging at an alarming rate in many parts of the world, especially in developing countries. As a result, inexpensive treatment regimens of gonorrhea in those countries have been rendered ineffective while efficacious ones are often unaffordable [3]. In Zambia, recommended treatment for Gonorrhea is Ciprofloxacin and the alternative treatment is Ceftriaxone [4]. Ciprofloxacin, a Fluoroquinolone antibiotic, was among the Centers for Disease Control and Prevention (CDC) recommended single-dose gonorrhea treatment regimens and is approximately half the cost of other CDC-recommended oral treatment regimens [5]. This could be one of the reasons why it is still used in developing countries like Zambia despite the rise in resistance. It is generally expected that a standard 
treatment regimen has to cure more than $95 \%$ of gonorrhea infections. This has been the acceptable threshold in the past. Therefore an antibiotic should not be administered to patients when more resistance levels of local isolates exceed 5\%. [6].

In gonorrhea, in vitro susceptibility to antibiotics simulates therapeutic outcome as the two are closely related. It is therefore possible to monitor resistance patterns in humans by monitoring susceptibility patterns in Neisseria gonorrhea isolates. This can be used to determine the prevalence of resistance and to detect any change in pattern at an early stage, thereby permitting standardized treatment regimens to be formulated for use both in developed and developing countries. Since antibiotic resistance affects our ability to treat and control gonorrhea, continuing surveillance is essential to monitor both its emergence and spread. Population-based surveillance of susceptibility patterns is necessary to establish standardized treatment regimens that cure at least $95 \%$ of infections and to modify the regimens when the situation changes. Effective surveillance requires both laboratory facilities and procedures capable of detecting resistance and the infrastructure for handling and analyzing data [7].

Managing the problem of antimicrobial resistance in developing countries should include the following, 1) Improved access to diagnostic laboratories, 2) Surveys to detect the emergence of resistance, 3) Regulation of the use of antibiotics, 4) Train prescribers of antibiotics, and 5) Education of the public [8]

According to Greenland [9], in the study of the dependence of a response variable on a set of independent variables, the choice of a model is largely determined by the scale of measurement of the response variable. When an outcome has two levels (e.g. dead or alive), binary logistic regression is commonly used to determine the association and its direction, between the outcome and all possible explanatory variables. Multinomial logistic regression similarly uses this approach but is applicable when instead there are more than two possible levels. Ordinal logistic regression is an extension of multinomial regression, which can be advantageous because it takes into account that the outcome variable has levels that have an ordering or can be termed as ordinal. Ordinal outcomes are common in medical research, but for the purposes of analysis it is often the case that such a variable will be recoded to just two levels, in order to be able to use standard binary logistic regression. For example, a pain score might be recoded to 'some pain' versus 'none', or 'severe pain' versus the rest. However, this strategy risks both data dredging and there is loss of valuable information in the data [10].

Correct estimation when using special statistical method will only be possible if a research question is addressed correctly, and the data comply with the assumptions required for that method. In this case our response variable is at 3 levels which are Sensitive, Intermediate and Resistant and therefore meeting the criteria for use of an Ordinal Logistic Regression as there are 3 categories with some inherent order.

\section{Methods}

2.1 Study Area 
The study was conducted at the University Teaching Hospital Adult Hospital (Sexually Transmitted Infections Laboratory) as well as the Women and Newborn Hospital (Gynae Ward). Patients at the University Teaching Hospital, that is, the Adult Hospital and Women and Newborn Hospital presenting with discharge and symptoms in line with Gonorrhea and gave consent between, were recruited into the study. A Questionnaire was administered and genital swabs were collected. The culturing and susceptibility testing was done at the STI lab and 104 isolates were obtained and Antimicrobial Susceptibility Testing using Ceftriaxone and Ciprofloxacin was performed.

\subsection{Study Variables}

The Independent variables were: Sex, Marital Status, Education, Occupation, Monthly Income, More than one Sexual Partner, Sexual Orientation, Ever Slept with a Sex Worker, Residence, Hospital, Drug and Agegroup. The outcome was Susceptibility and had three levels; Susceptible, Intermediate or Resistant with an assumed Ordinal nature.

The Objectives of this Study were: to identify and compare the resistance patterns of Gonorrhea to Ciprofloxacin and Ceftriaxone and to determine if drug resistance can be related to sex by comparing general resistance trends in men and women; to assess the socio-demographic, economic and behavioral characteristics associated with antimicrobial resistance; and to model the data using the Proportional Odds Model so as to explain the association between the susceptibility patterns and the independent variables.

\section{DATA MANAGEMENT AND ANALYSIS}

Data Analysis was done using STATA software. Fisher's Exact test was used to make summaries of the collected data. The Chi-Squared test for association was not used as all variables had some categories which had less than five observations.

The outcome measurement is continuous but was however categorized into the three possible results namely Susceptible, Intermediate and Resistant, which depends on the diameter of clearing measured from the agar plate, with reference to the set standard. Therefore, Ordinal Logistic Regression was used because the categories have an assumed ordering. Simple Ordinal logistic regression was run for each of the independent variables, that is, analysis predicting the ordinal dependent variable with each of the independent variables. This was also done with the view of checking whether the predictor variable contributes to the model or not.

A multiple ordinal logistic regression model was run to get adjusted estimates. The Brant Test was performed on the final model to check for the proportional odds assumption, with a significant omnibus test indicating that the proportional odds assumption was violated

\section{Results}

NOTE: Variables labelled a - i have missing values 
The variables Sex, Residence, Hospital, Drug and Age-group showed evidence of an association with the response variable (see Table 1 in the Supplementary Files). The variables more than one sexual partner and an individual's sexual orientation were also considered as Priori despite not having evidence of association, because these have been shown to predispose one to resistant strains of Neisseria gonorrhea.

REF- Reference category.

The variables sex of the participant from which sample was collected, the ward at the patient was seen and the drug inoculated as well as the agegroup 30 to 39 showed evidence of an effect with the susceptibility pattern in simple regression (as can be seen in Table 2 in the Supplementary Files). However at multiple regression, the association was not statistically significant for sex and ward indicating that confounding was present. This was adjusted for using stepwise investigator led regression.

Partial Proportional Odds model was used since the Proportional lines assumption was violated in the POM. In line with this, a model with constrains on all independent variables to meet the proportional odds/ parallel lines assumption was estimated.

Table 3 (in the Supplementary Files) shows that Males were 4.1 ( $95 \% \mathrm{Cl} ; 1.8,9.4$, p-value=0.001) times more likely to have Intermediate or Susceptible results compared to Resistance than females, or they were more likely to have Susceptible compared to Resistance or Intermediate result compared to females. Ciprofloxacin was $70 \%$ less likely than Ceftriaxone of having susceptible or intermediate results compared to resistance and this could be as high as $90 \%$ and as high as $40 \% \mathrm{p}$-values $<0.001$ ).

Upon testing parallel lines assumption using the .05 level of significance, Constraints for parallel lines imposed for drug gave a $p$-value $=0.995$ and Constraints for parallel lines imposed for sex gave a $p$-value $=0.554$. This showed that all explanatory variables in the final model meet the parallel lines assumption. Wald test of parallel lines assumption for the final model gave an insignificant test statistic (0.9549) which further indicated that the final model did not violate the proportional odds/ parallel lines assumption.

\section{Discussion}

The Partial Proportional Odds Model was used as the final model instead of Ordinal Logistic Regression with the variables Drug administered and Sex of participant.

The level of Ciprofloxacin resistance detected $(22.3 \%)$ is greater than the level (5\%) at which an alternative antibiotic regimen is recommended. This is consistent with studies conducted in other parts of the world as resistance to previously recommended ciprofloxacin and Ofloxacin regimens currently exceeds $40 \%$ in some Asian countries [7, 8].Substantial Quinolone resistance has also been identified only in South Africa, but ongoing systematic data collection and analysis are limited [7]. 
The level of resistance detected $(0 \%)$ indicates that for patients presenting with Gonorrhea at the University Teaching Hospital, Ceftriaxone combined with azithromycin is a satisfactory option for the first-line treatment of Gonorrhea.

Males were found to be less at risk of having antibiotic resistance as compared to females. And Ciprofloxacin was also less likely to cure Gonorrhea as compared to Ceftriaxone. This is in line with studies conducted in other parts of the world where Ciprofloxacin is no longer used for treatment because of high levels of resistance to it.

A study by Hailemariam indicated that some of the factors that may be associated with AMRNG were being male as compared to being females [3]. Jakopanec [11] came to the conclusion that men who have sex with men had a higher chance of getting AMRNG than heterosexual men. However the proportion of isolates from males $26(12.56 \%)$ that were tested was lower than those from females $181(87.44 \%)$ and this could have influenced the direction of the estimates.

Anti-Microbial Resistance surveillance is essential to optimize standard treatments is often lacking or of poor quality in countries with high disease rates. In Zambia, data on anti-microbial resistance in Neisseria gonorrhea is limited as Syndromic management of patients is practiced in medical facilities, which is that they are given a combination of Drugs for all possible STIs without a definitive laboratory diagnosis. This makes it difficult to have an active antimicrobial resistance surveillance system and have an efficient way of updating treatment guidelines as required. This study was therefore able to highlight the need to monitor resistance trends because the high level of resistance found (22.3\%), that we may be treating patients wrongly because we are giving them ineffective treatment. The paucity of updated data has made it difficult to understand of the extent and complexity of the problem with regard to our local circumstances. This cripples the prospect of putting up appropriate preventive and control intervention measures of antibiotic resistant $N$.gonorrhea, which could subsequently have negative consequences on the already pressured health service delivery system in Zambia.

The evaluation of the treatment algorithms could be undertaken every few years and assess costeffectiveness of the approach in areas of gonococcal resistance where more expensive second or thirdline drugs are given. More recently, the WHO has put in place strategies to intensify global AMR surveillance with emphasis on multi and extensively drug-resistant $N$ gonorrhoea surveillance, through revision of surveillance standards and describe use of a new panel of $N$ gonorrhea control strains. Addressing these new challenges posed by gonococcal AMR remain key in reducing the global burden of gonorrhea combined with implementation of wider strategies for general AMR control, and better understanding of mechanisms of emergence and spread of AMR [12].

\section{Conclusion}

The findings of this study indicate that antibiotic resistance is as much a reality in Zambia as it is in other parts of the world. The level of Ciprofloxacin resistance detected in this study $(22.3 \%)$ is greater than the level (5\%) at which an alternative antibiotic regimen is recommended. The level of resistance detected 
$(0 \%)$ indicates that for patients presenting with Gonorrhea at UTH, Ceftriaxone is a satisfactory option for first-line treatment of Gonorrhea. Women were found to be more likely to have resistance when infected with Gonorrhea in comparison with their male counterparts. The Partial proportional Odds model was used for final analysis of the predictors and the outcome.

\section{RECOMMENDATIONS}

Programs for continued surveillance and involvement of other hospitals in the surveillance program would give a better picture of resistance patterns on a wider scale. Future study's should include randomised controlled trials or utilising already available data to monitor trends and identify risk factors as primary data can be limiting in a study constrained by time. Also a shift from the use of Ciprofloxacin should be considered.

\section{Declarations}

\section{Ethical Approval and Consent to Participate}

Ethical clearance was obtained from University of Zambia Biomedical Ethics Research Committee (UNZABREC Ref \# 033-06-17) and permission to conduct the study was sought from the University Teaching Hospital's respective Senior Medical Superintendents and Heads of Departments. Written Consent from the participants was obtained so that the samples that had been collected could be analysed and also for the questionnaire to be administered. The specimens were de-identified in the laboratory once they are collected to protect the participants, by maintaining confidentiality. All questionnaires and data collection tools were submitted and approved by the UNZABREC (Ref \# 033-0617).

\section{Consent to Publish}

Not applicable

Availability of Data and Materials

Data sharing is not applicable to this article as no datasets were generated during the current study. However the data used for this study will be submitted to the University Teaching Hospital Sexually Transmitted Infections Laboratory, Lusaka, Zambia and can be accessed using relevant channels.

Competing Interest

The authors declare no conflict of interest, financial or otherwise.

Funding

Priscilla Kapombe's Master's Program was supported through the Development Excellence in Leadership, Training and Science (DELTAS) Africa Initiative Sub-Saharan Africa Consortium for Advanced 
Biostatistics (SSACAB) (Grant No.107754/Z/15/Z). The DELTAS Africa Initiative is an independent funding scheme of the African Academy of Sciences (AAS) Alliance for Accelerating Excellence in Science in Africa (AESA) and is supported by the New Partnership for Africa's Development Planning and Coordinating Agency (NEPAD Agency) with funding from the Welcome Trust (Grant No.107754/Z/15/Z) and the UK government. The views expressed in this publication are those of the authors and not necessarily those of the AAS, NEPAD Agency, Wellcome Trust or the UK government.

Authors Contributions

All authors contributed extensively to the work presented in this paper. All authors read and approved the final manuscript. Patrick Musonda gave overall guidance in the execution of study as well as analysis plan and methods. Lungowe Sitali also provided oversight in the appropriate Microbiological lab methods. Priscilla Kapombe conducted the study, performed analysis to arrive at the stated findings and wrote the manuscript under supervision of the other two co-authors.

Human and Animal Rights

No humans/ animals were used for the studies that are basis of this research.

Acknowledgements

Patrick Musonda would like to that some of his time is supported by the Research Council of Norway through its Centres of Excellence Scheme to the Centre of Intervention Science in Maternal and Child Health (CISMAC; project number 223269) and through the Global Health and Vaccination Programme (GLOBVAC; project number 248121). In addition, some of his time is also supported by the welcome trust; the Department for International Development; the Alliance for Accelerating Excellence in Science in Africa (DELTAS). Grant Number: [107754/Z/15/Z]. None of these organizations has contributed in any way in writing of this manuscript; any error arising in this publication is thoroughly the authors' problem.

\section{References}

1. World Health Organization, Department of Reproductive Health and Research. Global Action Plan To Control The Spread And Impact Of Antimicrobial Resistance in Neisseria gonorrhea. WHO, Geneva, Switzerland, 2012; pg 1-36 2. Gerbase AC, Global prevalence and incidence estimates of selected curable STDs. Sexually Transmitted Infections, 1998 Vol 74:1, S12-S16

[https://www.ncbi.nlm.nih.gov/pubmed/10023347] 3. Hailemariam M, Abebe T, Mihret A and Lambiyo T, Prevalence of $\mathrm{N}$. gonorrhoeae and their antimicrobial susceptibility patterns among symptomatic women attending gynaecology out patient department in Hawwasa Referal Hospital, Hawassa, Ethiopia. Ethiop J Health Sci, 2013; Vol 23:1 pg 10-7. [https://www.ncbi.nlm.nih.gov/pubmed/23559833] 4. Khalil G, Zambia HIV National Guidelines The Johns Hopkins University on behalf of the School of Medicine, Point of Care-Information Technology (POC-IT) Center and the Johns Hopkins University's international health care affiliate, 2008; Jhpiego Corporation 5. Burstein G, Stuart M, Berman JL and Blumer J, Ciprofloxacin 
for the Treatment of Uncomplicated Gonorrhea Infection in Adolescents: Does the Benefit Outweigh the Risk? Clinical Infectious Diseases, 2002; Vol 35; 2 pg S191-S199. 6. World Health Organization, Management of Sexually Transmitted Diseases, 1997; Rev.1. 7. Tapsall, J, Antimicrobial resistance in Neisseria gonorrhea. World Health Organization, 2001 http://www.who.int/drugresistance/Antimicrobial_resistance_in_Neisseria_gonorrhoeae.pdf. 8. HabteGabr E, Antimicrobial Resistance: A Global Public Health Threat. Journal of Eritrean Medical Associaton JEMA, 2010; Vol 1pg 36-40 [https://www.ajol.info/index.php/jema/article/download/49627/35956] 9. Greenland.S, An Application of Logistic Models to the Analysis of Ordinal Responses, Biometrical J, 1985; Vol 27 pg 189-197 [https://onlinelibrary.wiley.com/doi/abs/10.1002/bimj.4710270212] 10. Warner P, Ordinal logistic regression, J Fam Plann Reprod Health Care, 2008; Vol 34, pg 169-170. 11. Jakopanec I, Borgen K and Aavitsland P, The Epidemiology of Gonorrhea in Norway, 1993-2007: past victories, future challenges, BMC Infectious Diseases, 2009; Vol 9:33, pg 6.

[https://www.ncbi.nlm.nih.gov/pmc/articles/PMC2666741/] 12. Tapsall J, Ndowa F, David AL and Unemo $\mathrm{M}$, Meeting the public health challenge of multidrug- and extensively drug-resistant Neisseria gonorrhoeae, Expert Review of Anti-infective Therapy, 2009; Vol 7:7, pg 821-834

[https://www.ncbi.nlm.nih.gov/pubmed/19735224] 13. Norusis M, Ordinal regression Statistical Package for the Social Science (SPSS) 13.0 Advanced Statistical Procedures Companion, 2005; Chapter 4. 14. Heymans R, Bruisten SM, Golparian D, Unemo M, De Vries HJ and Van Dam AP, Clonally related Neisseria gonorrhea Isolates With Decreased Susceptibility To The Extended-Spectrum Cephalosporin Cefotaxime In Amsterdam, The Netherlands Antimicrob Agents Chemother, 2012; Vol 56, pg 1516-1522. [https://www.ncbi.nlm.nih.gov/pmc/articles/PMC3294943/] 15. Grad YH, Kirkcaldy RD, Trees D, Dordel J, Harris SR, Goldstein E, Weinstock H, Parkhill J, Hanage WP, Bentley S and Lipsitch M, Genomic Epidemiology Of Neisseria Gonorrhoeae With Reduced Susceptibility To Cefixime In The USA: A Retrospective Observational Study. Lancet Infect Dis, 2014; Vol 14, pg 220-226.

[https://www.ncbi.nlm.nih.gov/pubmed?linkname=pubmed_pubmed\&from_uid=27638945] 16. Brant, R, Assessing proportionality in the proportional odds model for ordinal logistic regression. Biometrics, 1990; Vol 46, 1171-1178. [https://www.ncbi.nlm.nih.gov/pubmed/2085632] 17. Allison, P. D, Logistic regression using the SAS system: Theory and application. Cary, NC, 1999; SAS Institute. 18. Clogg, C., \& Shihadeh, E. S, Statistical models for ordinal variables, 1994; Thousand Oaks, California: Sage publications. 19. O'Connell, A, Logistic regression models for ordinal response variables, 2006; Thousand Oaks: Sage publications. 20. World Health Organization, Western Pacific Region Gonococcal Antimicrobial Susceptibility Programme (GASP) report,Commun. Dis. Intell, 2001; Vol 25, pg 274-277. 21. Arising From Mathematical Modeling, The Journal of Infectious Diseases, Vol 213:6, pg 883-890 22. SAS Technical Report P-200, SAS/STAT software: CALIS and Logistic procedures and BMDP statistical software manual, 1990; Vol 2, Berkeley University Press. 23. Shan G and Changxing Ma, Unconditional tests for comparing two ordered multinomials, Statistical Methods in Medical Research, 2012; Vol 25:1, pg 241 254. 24. Supriya DM, Maclean I, Ndinya-Acholaj O, Moses S, Martin I, Ronald A, Agunda L, Murugu R, Bailey RC, Melendez R and Zenilman J, Emergence of Quinolone Resistance and Cephalosporin MIC Creep in Neisseria gonorrhea Isolates from a Cohort of Young Men in Kisumu, Kenya, 2002 to 2009.Antimicrobial Agents And Chemotherapy, 2011; Vol 55:8 pg 3882-3888. 


\section{Tables}

Due to technical limitations, tables are only available as downloads in the supplemental files section.

\section{Supplementary Files}

This is a list of supplementary files associated with this preprint. Click to download.

- Table2.jpg

- Table1.jpg

- Table3.jpg 